\title{
Functionally Independent Columns of Rat Somatosensory Barrel Cortex Revealed with Voltage-Sensitive Dye Imaging
}

\author{
Carl C. H. Petersen and Bert Sakmann \\ Department of Cell Physiology, Max-Planck-Institute for Medical Research, Heidelberg D-69120, Germany
}

\begin{abstract}
Whisker movement is somatotopically represented in rodent neocortex by electrical activity in clearly defined barrels, which can be visualized in living brain slices. The functional architecture of this part of the cortex can thus be mapped in vitro with respect to its physiological input and compared with its anatomical architecture. The spatial extent of excitation was measured at high temporal resolution by imaging optical signals from voltage-sensitive dye evoked by stimulation of individual barrels in layer 4 . The optical signals correlated closely with subthreshold EPSPs recorded simultaneously from excitatory neurons in layer 4 and layer 2/3, respectively. Excitation was initially $(<2 \mathrm{msec})$ limited to the stimulated barrel and subsequently (>3 msec) spread in a columnar manner into layer 2/3 and then subsided in both layers after $\sim 50 \mathrm{msec}$. The lateral extent of the response was limited to the cortical column defined structurally by the barrel in layer 4. Two experimental
\end{abstract}

interventions increased the spread of excitation. First, blocking $\mathrm{GABA}_{\mathrm{A}}$ receptor-mediated synaptic inhibition caused excitation to spread laterally throughout wide regions of layer $2 / 3$ and layer 5 but not into neighboring barrels, suggesting that the local excitatory connections within layer 4 are restricted to single barrels and that inhibitory neurons control spread in supragranular and infragranular layers. Second, NMDA receptor-dependent increase of the spread of excitation was induced by pairing repetitive stimulation of a barrel column with coincident stimulation of layer $2 / 3$ in a neighboring column. Such plasticity in the spatial extent of excitation in a barrel column could underlie changes in cortical map structure induced by alterations of sensory experience.

Key words: neocortex; somatosensory cortex; barrel cortex; imaging; voltage-sensitive dye; plasticity
The rodent somatosensory barrel cortex provides a unique opportunity to identify specific cortical locations in brain slice preparations. Each whisker is represented in the neocortex by a barrel that can be visualized at high resolution in the living brain slice (Woolsey and Van der Loos, 1970; Agmon and Connors, 1991; Petersen and Sakmann, 2000). The local neocortical neuronal network can thus be investigated in vitro within the context of a well defined somatotopic map. A comparison of data obtained from in vitro and in vivo recordings may delineate the exact contribution of different neuronal ensembles to the in vivo response.

The spatiotemporal structure of whisker-evoked responses was initially inferred by repeated measurements of extracellular unit recordings (Armstrong-James et al., 1992). These measurements suggested that cortical responses to single whisker deflections were initiated in a single barrel in layer 4 and then spread vertically in a columnar manner into layer $2 / 3$. At longer latencies, excitation was recorded in neighboring columns. Simultaneous recordings from multiple sites over the barrel cortex region with a multielectrode array confirmed the initial excitation of a single barrel, followed by spread to neighboring barrels (Petersen and Diamond, 2000). The spatial resolution of extracellular unit recordings is low, and they do not measure subthreshold responses. Higher spatial resolution (but without temporal resolution) single whisker responses has been obtained from 2-deoxyglucose uptake

Received June 1, 2001; revised Aug. 6, 2001; accepted Aug. 9, 2001.

C.C.H.P. was supported by a Marie Curie fellowship from the European Commission. We thank Dr. Asaf Keller for critical reading of an earlier version of this manuscript and Dr. Amiram Grinvald for technical help and useful discussions.

Correspondence should be addressed to Carl C. H. Petersen, Department of Cell Physiology, Max-Planck-Institute for Medical Research, Jahnstrasse 29, Heidelberg D-69120, Germany. E-mail: petersen@mpimf-heidelberg.mpg.de.

Copyright (C) 2001 Society for Neuroscience $\quad 0270-6474 / 01 / 218435-12 \$ 15.00 / 0$ studies (Durham and Woolsey, 1977; Kossut et al., 1988; McCasland and Woolsey, 1988) and optical imaging of intrinsic reflectance changes of the cortical surface (Masino and Frostig, 1996). An alternative approach is to image neuronal activity with voltage-sensitive dyes, which can record both the spatial and temporal structure of layer $2 / 3$ responses with high resolution. In such optical recordings, an even larger area of cortex appears to be excited by stimulation of a single whisker (Orbach et al., 1985; Kleinfeld and Delaney, 1996). Large single whisker subthreshold receptive fields have also been reported with whole-cell voltage recordings from neurons in layers 2-5 (Moore and Nelson, 1998; Zhu and Connors, 1999). The functional response of a large area of neocortex after single whisker stimulation appears to be at odds with the apparent anatomical precision of the wiring from single whiskers to single layer 4 barrels.

To investigate the spatiotemporal dynamics of neocortical responses under more controlled conditions, we stimulated single barrels in vitro and imaged voltage-sensitive dye signals at high resolution. Unlike responses to single whiskers in vivo, stimulation of a single barrel evoked a response primarily limited to the width of a single barrel. The spread of excitation, although invariant under many manipulations, could however be expanded by blockade of $\mathrm{GABA}_{\mathrm{A}}$-mediated inhibition. The lateral extent of layer 2/3 responses could also be expanded in an NMDA receptor-dependent manner by pairing stimulation of a barrel column with stimulation of layer $2 / 3$ in a neighboring column, a process that could underlie map plasticity induced by alterations in sensory experience.

\section{MATERIALS AND METHODS}

Preparation of slices of somatosensory barrel cortex. Wistar rats (13-15 d old) were decapitated under deep halothane anesthesia. The brain was 
rapidly removed and placed in ice-cold extracellular medium. One of three types of slices was subsequently prepared. The majority of experiments were performed on 350- $\mu \mathrm{m}$-thick thalamocortical slices prepared following the description of Agmon and Connors (1991), with modifications (Petersen and Sakmann, 2000). Other experiments were performed on $350-\mu \mathrm{m}$-thick slices cut to reveal the five rows (A-E) of the posterior medial barrel subfield following the description of Finnerty et al. (1999). The response properties of these two types of paracoronal slices were not different. Additionally, some experiments were performed on $250-\mu \mathrm{m}$ thick slices cut tangential to the pia at a depth such that the slices contained layer 4 barrels as described by Fleidervish et al. (1998). Slices were cut by a vibratome in ice-cold extracellular medium and were subsequently incubated at $35^{\circ} \mathrm{C}$ for $15-30 \mathrm{~min}$ after slicing. The slices were then transferred to room temperature $\left(20-23^{\circ} \mathrm{C}\right)$ until required for analysis. Throughout the procedure, slices were maintained in extracellular medium containing (in mM): $125 \mathrm{NaCl}, 25 \mathrm{NaHCO}_{3}, 25$ glucose, 2.5 $\mathrm{KCl}, 1.25 \mathrm{NaH}_{2} \mathrm{PO}_{4}, 2 \mathrm{CaCl}_{2}$, and $1 \mathrm{MgCl}_{2}$ (bubbled with $95 \% \mathrm{O}_{2}$ and $5 \% \mathrm{CO}_{2}$ ).

Optical recording of voltage-sensitive dye signals. Slices were incubated at room temperature in extracellular medium with $0.1 \mathrm{mg} / \mathrm{ml} \mathrm{RH} 155$ (Molecular Probes, Eugene, OR) for 15-30 min as described by Laaris et al. (2000). Slices were washed for $15 \mathrm{~min}$ in the experimental chamber before use to remove unbound dye. All experiments were performed at $35^{\circ} \mathrm{C}$. Glass microelectrodes (similar to those used for whole-cell patchclamp recordings, although slightly larger in tip diameter) were filled with extracellular medium to form an extracellular stimulating electrode. Constant amplitude current pulses (200 $\mu$ sec, $10-100 \mu \mathrm{A})$ were delivered with an A365 stimulus isolator (World Precision Instruments, Sarasota, FL). For slices cut normal to the pia, the tip of the stimulating electrode was placed at the bottom of layer 4 in the center of a visually identified barrel. For tangential slices, the stimulating electrode was placed in the middle of a barrel. The slice was illuminated with light of $700 \pm 25 \mathrm{~nm}$ wavelength (Omega Optical, Brattleboro, VT) and viewed with a Zeiss $10 \times$ water immersion lens giving a field of view of $775 \mu \mathrm{m}$. Quantitative optical recordings of changes in light transmission were made with the Deltaron HR1700 (Fuji, Tokyo, Japan), which consists of an array of $128 \times 128$ detectors; thus, each pixel receives light from a $6 \times 6 \mu \mathrm{m}$ region of the slice. In experiments of the type shown in Figures 8 and 9 , a larger field of view was imaged by using a $0.5 \times \mathrm{C}$-mount adaptor, and, in these cases, each pixel received light from a region of $12 \times 12 \mu \mathrm{m}$. Evoked changes in light transmission measured with this voltagesensitive dye at low stimulation intensities were $<0.1 \%$. These small changes in light intensity could be well resolved with a time resolution of $0.6 \mathrm{msec}$ per frame with the differential gain of the Deltaron HR1700 camera set in the range between 60 and 1000. To reduce noise, 16 consecutive stimuli were averaged with each stimulus separated by an interval of $5 \mathrm{sec}$. Image data were analyzed off-line by custom-written routines in IgorPro (WaveMetrics Inc., Lake Oswego, OR). Differential images were divided by the prestimulus image, and voltage-sensitive dye signals are thus presented as fractional changes in light intensity.

Whole-cell recording of membrane potential. Simultaneous whole-cell patch-clamp recordings were established with excitatory neurons of layer 2/3 and layer 4 using Axopatch 200 amplifiers (Axon Instruments, Foster City, CA). Whole-cell recordings were established using pipettes with resistances of $5 \mathrm{M} \Omega$ filled with a solution containing (in $\mathrm{mM}$ ): 130 potassium gluconate, 10 sodium gluconate, 10 HEPES, 10 phosphocreatine, $4 \mathrm{NaCl}, 4 \mathrm{MgATP}$, and $0.3 \mathrm{Na}_{3} \mathrm{GTP}$, adjusted to $\mathrm{pH} 7.2$ with $\mathrm{KOH}$. Biocytin $(3 \mathrm{mg} / \mathrm{ml})$ was routinely included in the intracellular solution. Excitatory neurons in layer $2 / 3$ were easily distinguished by the prominent apical dendrite and pyramidal shape of somata. Within layer 4 , excitatory neurons had small round somata and were often found in clusters. These neurons were further identified as excitatory based on their regular firing patterns to depolarizing current and broad action potentials (APs) $(\sim 1 \mathrm{msec}$ half-width). Electrophysiological data were collected simultaneously with the optical voltage-sensitive dye imaging, and thus 16 consecutive sweeps of extracellular stimulation were also averaged for these electrical recordings. Data were analyzed and aligned with optical recordings using custom written routines in IgorPro.

Morphological reconstruction of biocytin stained neurons. After filling neurons with biocytin, slices were fixed overnight at $4{ }^{\circ} \mathrm{C}$ in $4 \%$ paraformaldehyde. The slices were washed with PBS (100 mM sodium phosphate, $\mathrm{pH}$ 7.2) five times over a period of $2 \mathrm{hr}$. Endogenous peroxidases were then quenched by a 5 min incubation with $1 \% \mathrm{H}_{2} \mathrm{O}_{2}$. The slices were subsequently rinsed in PBS five times over a period of $2 \mathrm{hr}$. Slices were conjugated with avidin-biotinylated horseradish peroxidase following the instructions of the manufacturer (ABC-Elite, Vector stains; Vector Laboratories, Burlingame, CA). Slices were then washed five times over a period of $2 \mathrm{hr}$ with PBS, and subsequently biocytin-stained neurons were visualized under a reaction with $0.5 \mathrm{mg} / \mathrm{ml} \mathrm{DAB}$ and $0.01 \% \mathrm{H}_{2} \mathrm{O}_{2}$. When the neuronal processes were clearly visible, the reaction was stopped by washing with PBS. Finally, the slices were mounted on slides using mowiol. Axonal and dendritic processes were subsequently reconstructed in three dimensions using Neurolucida software (MicroBrightField Inc., Colchester, VT).

\section{RESULTS}

\section{Voltage-sensitive dye signals report the spatiotemporal pattern of subthreshold EPSPs}

Simultaneous recording of voltage-sensitive dye signals and whole-cell membrane potential

Field stimulation with a large-diameter patch electrode filled with extracellular solution placed in the lower center of a layer 4 barrel (Fig. 1A) evoked responses that could be detected by both voltage-sensitive dye imaging and membrane potential recordings from excitatory neurons. Slices were stained with the voltagesensitive dye RH155, which inserts into the outer leaflet of cell membranes and changes the degree of absorption of light at 700 $\mathrm{nm}$ depending on the potential across the membrane in which it is attached. The camera thus detects a bright-field image of the slice (Fig. $1 A$ ) on which small fractional changes in transmission of the order of $0.1 \%$ are spatially resolved in response to the field stimulus (Fig. 1D). Each pixel in the camera collected light from a region of $6 \times 6 \mu \mathrm{m}$, and image frames were captured at $0.6 \mathrm{msec}$ intervals (Fig. $1 F$ ). The time course and relative amplitudes of voltage-sensitive dye signals quantified in regions of $50 \times 50 \mu \mathrm{m}$ around the whole-cell recording electrodes in layer $2 / 3$ and layer 4 were similar to the evoked subthreshold EPSPs (Fig. 1E). Biocytin was routinely included in the whole-cell pipette solution to identify the dendritic and axonal arborizations of the layer 4 and layer $2 / 3$ excitatory neurons in the context of the layer 4 barrel structure (Fig. $1 B$ ) and reconstructed in three dimensions (Fig. 1C).

\section{Pharmacological characterization of voltage-sensitive dye signals}

In the absence of voltage-sensitive dye, no changes in light transmission could be evoked; thus, the entire recorded signal is attributable to the response of the voltage-sensitive dye RH155. No phototoxicity was apparent with the use of the dye at the concentrations and illumination intensities used in this study as assessed by resting membrane potential, AP discharge patterns, and membrane potential changes evoked by field stimulation. Optically detected signals were blocked by $1 \mu \mathrm{M}$ TTX bath application (normalized signal amplitude at $12 \mathrm{msec}$ was $-0.04 \pm$ $0.04 ; n=8$ ), suggesting that the voltage-sensitive dye signals are entirely dependent on AP generation and conduction. Removal of extracellular $\mathrm{Ca}^{2+}$ or blockade of glutamatergic synaptic transmission by $10 \mu \mathrm{M}$ NBQX and $100 \mu \mathrm{M}$ D-APV reduced the peak signal amplitude quantified across the entire field of view to similar extents $[0.22 \pm 0.08(n=8)$ for NBQX and APV; $0.21 \pm$ $0.08(n=8)$ for nominally $\mathrm{Ca}^{2+}$-free artificial CSF]. Thus, $>80 \%$ of the voltage-sensitive dye signal is attributable to the action of synaptically released glutamate (Yuste et al., 1997; Higashi et al., 1999; Laaris et al., 2000). The remaining $20 \%$ of the signal had a time course similar to that without pharmacological manipulation and presumably reflected direct activation of neuronal membrane near the stimulating electrode. Whole-cell voltage recordings made simultaneously suggested that the stimulation artifact was 
A

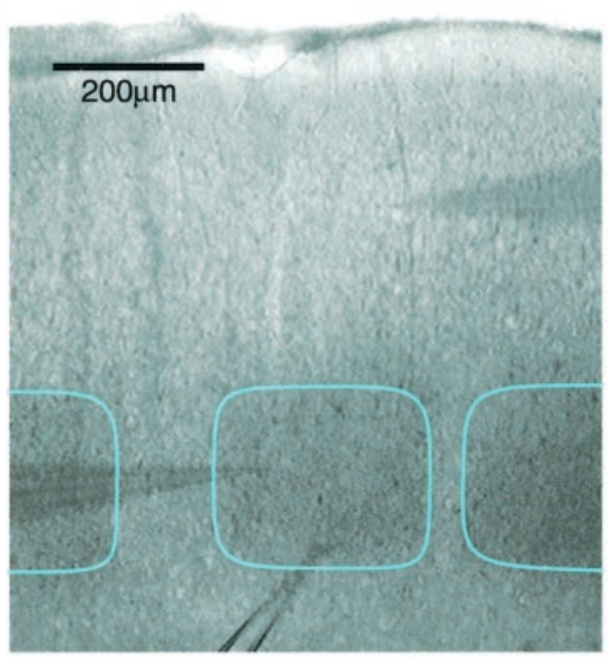

B

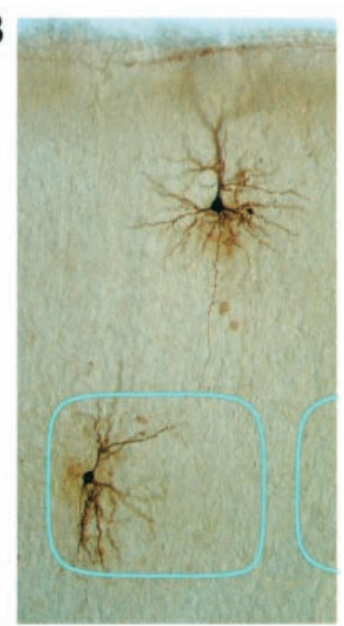

$0.1 \mathrm{E}$

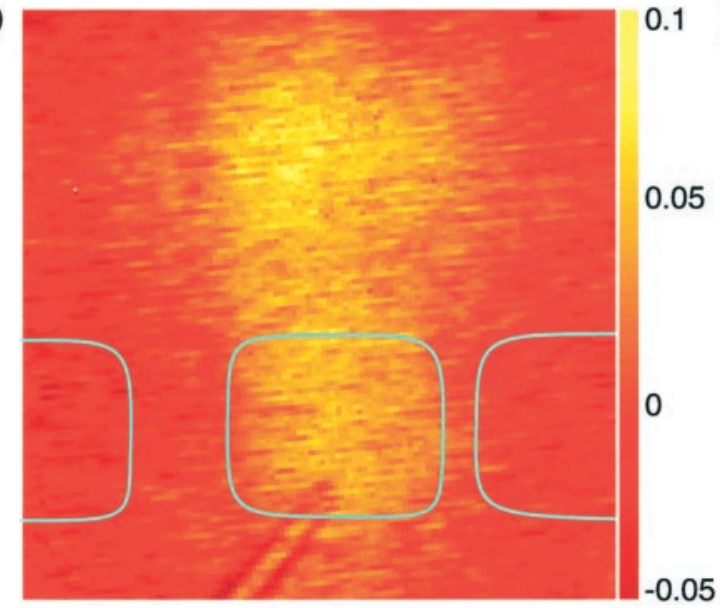

C

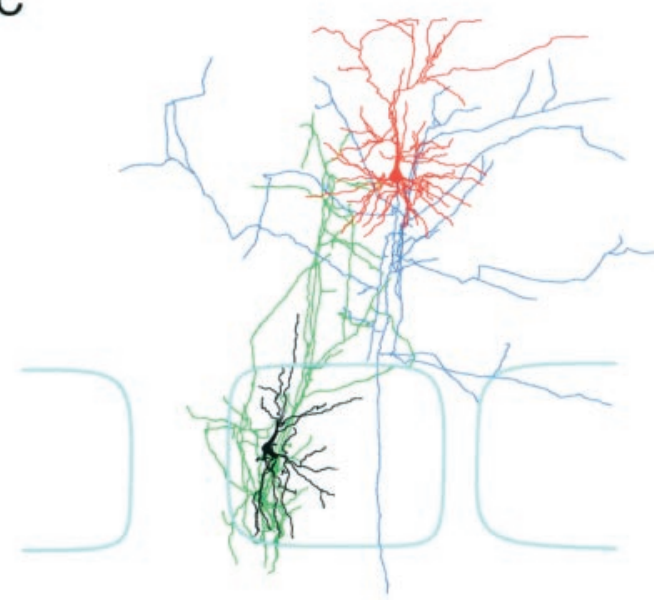

$\mathrm{F}$

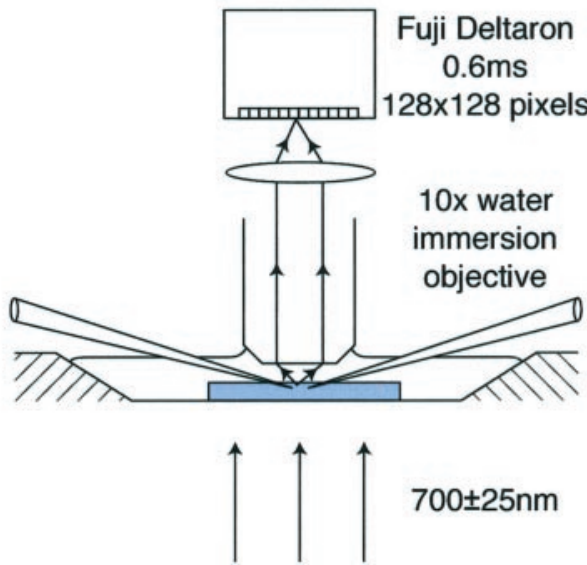

Figure 1. Experimental setup for simultaneous whole-cell and voltage-sensitive dye recording. $A$, Bright-field image of somatosensory barrel cortex from a slice stained with RH155. Dark regions correspond to the layer 4 barrels, which are outlined in cyan. Two whole-cell recording pipettes are visible, one in layer $2 / 3$ and the other in layer 4 . A large-diameter patch pipette filled with extracellular solution is used for field stimulation, with the tip positioned at the bottom of the central barrel. $B$, The excitatory neurons are filled with biocytin during the whole-cell recordings, allowing their structure to be visualized after fixation and staining. $C$, Reconstruction of the biocytin-filled layer $2 / 3$ pyramidal neuron (red dendrite and blue axon) and the layer 4 spiny stellate (black dendrite and green axon). $D$, A single unfiltered differential image normalized to the bright-field transmitted light $\left(\Delta I / I_{0}\right)$ of the voltage-sensitive dye signal taken $12 \mathrm{msec}$ after stimulation. E, Time course of voltage-sensitive dye responses (top traces) compared with the simultaneously obtained whole-cell recordings (bottom traces). The voltage-sensitive dye signals were quantified from an area of $50 \times 50 \mu \mathrm{m}$ around the location of the neurons from which the whole-cell recordings are made (layer 2/3 red traces and layer 4 blue traces). $F$, Optical arrangement for simultaneous whole-cell recordings and voltage-sensitive dye recordings. The slice is illuminated with $700 \mathrm{~nm}$ light from a halogen lamp and viewed with a $10 \times$ water immersion lens. The slice is stained with voltage-sensitive dye RH155, which increases its absorption of $700 \mathrm{~nm}$ light when membranes are depolarized. Small changes in the amount of light transmitted through the slice can be recorded with a Fuji Deltaron camera. With a square field of view of $775 \mu \mathrm{m}$ obtained under these conditions, each pixel covers a region of $6 \times 6 \mu \mathrm{m}$, and each frame has $0.6 \mathrm{msec}$ duration.

strongly enhanced during blockade of glutamatergic transmission, showing a component with a decay time course similar to evoked EPSPs. The larger stimulation artifact in the presence of NBQX and APV presumably results from the increased membrane resistance attributable to block of glutamate receptors, thus giving larger responses to the extracellular current injection. Removal of divalent cations increases membrane excitability, and such an effect may also account for the surprisingly large signal remaining in nominally $\mathrm{Ca}^{2+}$-free medium. The changes in membrane excitability complicate the pharmacological dissection of the voltage-sensitive dye signal but give the lower bound estimate that at least $80 \%$ of the dye signal is caused by synaptically released glutamate acting at ionotropic receptors and generating dendritic EPSPs. In support of a major postsynaptic component to voltagesensitive dye signals, Laaris et al. (2000) found that the removal of extracellular calcium was able to completely block neocortical signals evoked by stimulation of the thalamus, with the remote stimulation site in these experiments preventing direct activation of dendrites

\section{Anatomy of a "normalized barrel"}

Field stimulation is thought to result in the preferential excitation of axons rather than dendrites. To gain an understanding of the anatomy of some of the stimulated fibers, the axonal and dendritic arborizations of 18 layer $2 / 3$ and layer 4 neurons were reconstructed within the context of the borders of a barrel in layer 4 . To compare the arborizations of neurons from different experiments, both the horizontal and vertical dimensions were normalized. The horizontal widths of the layer 4 barrels were normalized to each other (mean barrel width, $310 \mu \mathrm{m}$; range, 170-435 $\mu \mathrm{m}$ ), but 
A

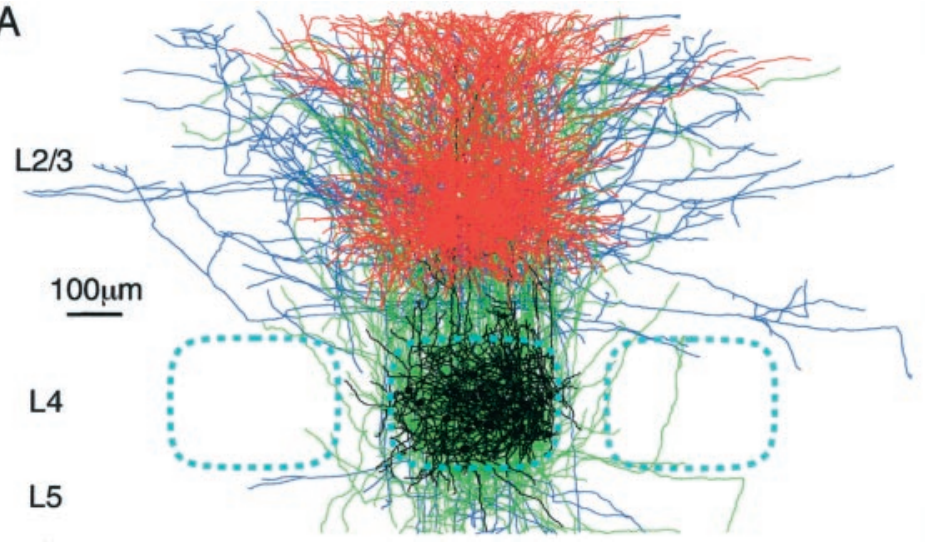

$\mathrm{B}$

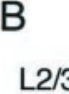

L2/3
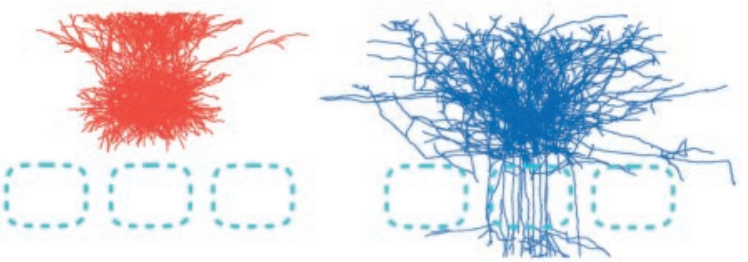

L5
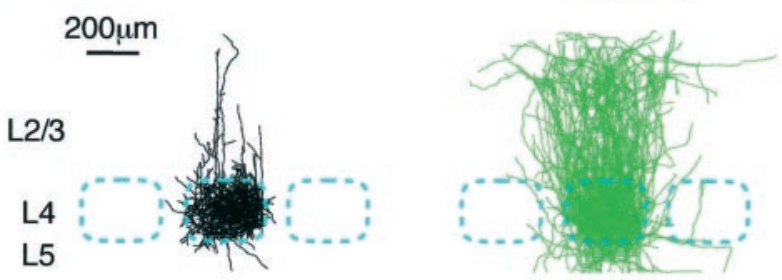

Figure 2. Morphology of excitatory neurons of layer $2 / 3$ and layer 4 within a barrel column. $A$, Excitatory neurons were filled with biocytin during whole-cell recordings and subsequently stained. Axonal and dendritic arbors were reconstructed in three dimensions for 18 layer $2 / 3$ and 18 layer 4 excitatory neurons. Two-dimensional projections into the original plane of the slice are illustrated from the pia to a depth including layer 5A. The dimensions of the reconstruction of each neuron are normalized with respect to the pia, bottom of layer 4 , and the lateral width of the layer 4 barrel, thus generating a representation of a normalized barrel column. Layer 2/3 pyramidal neurons are shown with red dendrite and blue axon. Layer 4 spiny stellate neurons are shown with black dendrite and green axon. The scale bar represents the mean normalization length. $B$, The same reconstructed neurons are shown with the dendritic and axonal compartments separated. The layer 4 dendrites are primarily confined to the layer 4 barrel. The layer 4 axon forms an ascending column of input to the layer $2 / 3$ pyramidal neurons. The major lateral spread of neuronal arborization derives from the axons of the layer $2 / 3$ pyramids.

the relative positions of the neuronal somata and barrel boundaries were maintained. The vertical dimensions were normalized using the pia and the layer $4 / 5$ boundary as the fixed points (mean distance from pia to layer 4/5 boundary, $810 \mu \mathrm{m}$; range, 735-900 $\mu \mathrm{m})$. The normalized reconstructions of 18 excitatory layer $2 / 3$ neurons and 18 excitatory layer 4 neurons were superimposed in the context of the barrel boundaries (Fig. 2). A field stimulus at the base of a layer 4 barrel is likely to primarily activate thalamocortical axons and axons of the layer 4 neurons, which form a very dense network in this region. The axons from pyramidal neurons in layer $2 / 3$ and layer $5 / 6$ are present at a much lower density at the base of a layer 4 barrel and are likely to make only a small contribution to evoked responses. Previous studies have shown that individual thalamocortical axons primarily innervate single layer 4 barrels (Jensen and Killackey, 1987). The axonal arborization of layer 4 spiny stellate and star pyramidal neurons is likewise primarily horizontally confined to individual barrels (Harris and Woolsey, 1983; Lorente de Nó, 1992; Bernardo et al., 1990; Lübke et al., 2000; Petersen and Sakmann, 2000). The response observed at the shortest latencies in both voltage-sensitive dye recordings and whole-cell recordings (Figs. 1,3) is thus likely to result from the postsynaptic action of glutamate released from thalamocortical and layer 4 axons acting on ionotropic glutamate receptors located on the dendrites of layer 4 neurons. Each layer 4 axon forms excitatory connections with approximately one-third of the excitatory neurons within its home barrel, with a mean EPSP amplitude of $\sim 1 \mathrm{mV}$ (Petersen and Sakmann, 2000). It would thus appear that, to evoke EPSPs averaging $\sim 5 \mathrm{mV}$ (Figs. $1,3)$ in the excitatory layer 4 neuronal network, APs are required in $<20$ presynaptic neurons. An AP with an amplitude of $\sim 100$ $\mathrm{mV}$ and a duration of $\sim 1 \mathrm{msec}$ can only give a small signal compared with that resulting from the released glutamate of the same AP, which evokes a mean depolarization of $\sim 1 \mathrm{mV}$ in one-third of the several thousand neighboring layer 4 neurons and lasting $\sim 50 \mathrm{msec}$. From such calculations, it becomes apparent why the voltage-sensitive dye signal predominantly reports subthreshold membrane depolarization rather than evoked APs.

The layer 4 axons project in a columnar manner into layer $2 / 3$
(Lübke et al., 2000), defining a "barrel column" and forming excitatory contacts predominantly onto the basal dendrites of pyramidal neurons (Feldmeyer et al., 1999). The axonal arborizations of these layer $2 / 3$ pyramidal neurons are not confined to the barrel column structure, and although the densest axonal arborizations are close to the soma, there are numerous collaterals that spread horizontally for long distances (Fig. 2). Depending on the degree of functional activation of these layer $2 / 3$ pyramidal neurons, they could propagate signals across different barrel columns. These anatomical reconstructions give a static representation of the excitatory neuronal network with which we can compare the functional spatiotemporal spread of excitation monitored with voltage-sensitive dye imaging. Not included in this anatomical reconstruction of a "normalized barrel" are inhibitory neurons, which are likely to regulate the degree of activity of the excitatory neurons. The voltage-sensitive dye signals will thus differ in at least two respects from the anatomical representation. First, they will report the time course of excitation in the neuronal network, and second, the voltage-sensitive dye signal will include the effects of inhibition. Furthermore, the anatomical reconstruction of the barrel column is deduced from a selected population of neurons from which whole-cell recordings were made. It is unknown how well these neurons represent the ensemble local neuronal networks within the entire depth of the slice from which the voltage-sensitive dye signal is derived.

\section{Spatiotemporal correlation of optical signals with simultaneous whole-cell membrane potentials}

Stimulation of a slice at the lower center of a layer 4 barrel evoked voltage-sensitive dye signals initiated in layer 4 , which subsequently spread to layer $2 / 3$ (Fig. 3). To correlate the time course and amplitude of the optically detected signals, dual whole-cell recordings were made simultaneously from excitatory neurons in layer 4 and layer 2/3, respectively. Coincident with the current injection $(200 \mu \mathrm{sec}, 10-100 \mu \mathrm{A})$, a small stimulation artifact appeared in both electrical and optical signals. This signal was small and of very short duration (a single frame of $0.6 \mathrm{msec}$ duration in the imaging) and was $\sim 0.5$ msec duration in whole- 

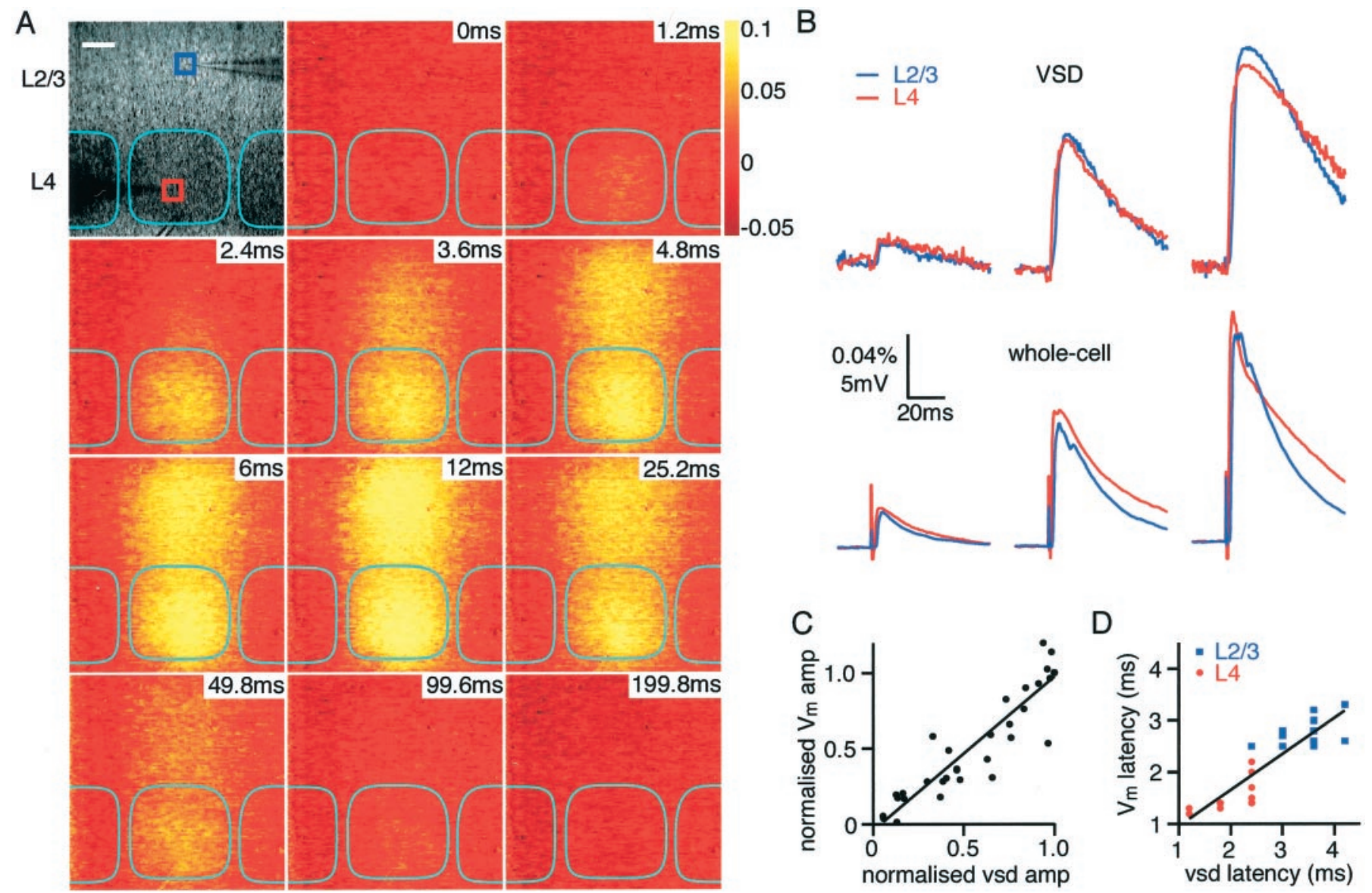

Figure 3. Correlation of voltage-sensitive dye signal with dual whole-cell recordings of membrane potential. $A$, Bright-field image of somatosensory barrel cortex from a slice stained with RH155. Dark regions correspond to the barrels. Two whole-cell recording pipettes are visible, one in layer 2 (blue box) and one in layer 4 (red box). The extracellular stimulation electrode is visible at the bottom of the micrograph at the border of layer 4 and 5 in the middle of a barrel. The time series of pictures indicates the optical response of the voltage-sensitive dye after stimulation at 0 msec. The images were collected with $0.6 \mathrm{msec}$ exposure time and are presented without filtering or averaging. Excitation can be observed to spread gradually from layer 4 to layer 2/3, maintaining a strict columnar excitation. Scale bar, $100 \mu \mathrm{m}$. B. Comparison of optical responses quantified from the $60 \times 60 \mu \mathrm{m}$ boxes indicated in $A$. Blue, layer 2/3; red, layer 4, with the membrane potential response of two individual neurons located within the boxes from which simultaneous whole-cell recordings were made. Three stimulus strengths are compared. As stimulus strength is increased, both the optical and the whole-cell responses increase in amplitude in a similar manner. Equally, the latency of responses in both layer 2/3 and layer 4 is similar in optical and whole-cell recordings. The decay kinetics are also similar, although in this example the voltage-sensitive dye signal decays somewhat slower. $C$, The amplitude of responses to the highest stimulation strength in an experiment was used to normalize the response to less intense stimuli and the relative response amplitude detected optically in a region $60 \times 60 \mu \mathrm{m}$ surrounding the soma of neurons from which the whole-cell recordings were made. The linear fit suggests a good correlation between the amplitudes of voltage signals detected optically and by whole-cell recordings. $D$, The latency to half-maximal response of recordings is greater in layer 2/3 (blue squares) than in layer 4 (red circles), as measured both optically and by whole-cell recordings. The dye latency, however, is somewhat slower than the latency determined by whole-cell recordings.

cell voltage recordings. The stimulus signal was also highly localized in the imaging experiments to within an $\sim 50 \mu \mathrm{m}$ radius of the stimulating electrode. After a latency of $\sim 1 \mathrm{msec}$, both optical and membrane voltage of layer 4 neurons revealed depolarizing signals. The latency for half-maximal responses in layer 4 was $2.0 \pm 0.2 \mathrm{msec}(n=10)$ measured optically, whereas the latency was $1.5 \pm 0.1 \mathrm{msec}(n=10)$ for whole-cell voltage recordings. The voltage-sensitive dye signal subsequently propagated vertically into layer $2 / 3$, and EPSPs were simultaneously observed in voltage recordings of layer $2 / 3$ pyramidal neurons. The latency to the half-maximal optical signal was $3.4 \pm 0.1 \mathrm{msec}(n=10)$ compared with $2.8 \pm 0.1 \mathrm{msec}(n=10)$ in voltage recordings. The peak amplitudes of voltage-sensitive dye and membrane potential signals were similar in layer $2 / 3$ and in layer 4 . By varying the stimulation strength, we could correlate the changes in voltagesensitive dye signal and evoked EPSPs. The amplitudes changed in a linearly correlated manner both comparing layer 4 and layer
$2 / 3$ and comparing optical and whole-cell voltage recordings (Fig. $3 B)$. Equally, the decay phase of optical and whole-cell voltage recordings appeared qualitatively similar [in some experiments, the voltage-sensitive dye signal decayed more slowly (Fig. 3B), but in other experiments the electrical response decayed more slowly (see Fig. 6B)]. The normalized correlation of the peak amplitude of signals recorded in all experiments between optical and voltage recording (Fig. $3 C$ ) shows a slope very close to unity $[\mathrm{wc}=1.02$ *vsd $-0.04 ; r^{2}=0.87 ; n=10$ (wc indicates whole-cell recording, and vsd indicates voltage-sensitive dye signal)]. The latency correlation (Fig. 3D) also appears to be linear, although the dye signal latency was longer ( $\mathrm{wc}=0.70 *$ vsd $+0.26 ; r^{2}=$ $0.77 ; n=10)$.

These correlations between the time course and amplitude of the two types of signals appear remarkably close considering that the neurons from which the whole-cell recordings were made represent a minute fraction of the population of the cell mem- 
brane area from which the voltage-sensitive dye signals are derived. The voltage-sensitive dye signal is thus able to resolve the spatiotemporal structure of small subthreshold EPSPs in ensembles of many neurons evoked by field stimulation.

\section{Spatial extent of voltage-sensitive dye signals in relationship to anatomical barrel boundaries}

Barrel boundaries can be visualized at high resolution in living brain slices (Petersen and Sakmann, 2000), allowing the spatial extent of the voltage-sensitive dye signal to be analyzed with reference to the anatomy of barrel cortex. From Figures 1 and 3 it is clear that the predominant spread of excitation is limited horizontally to the width of the layer 4 barrel, which is clearly distinguishable in the bright-field image. To analyze the lateral spread of excitation with respect to barrel boundaries more rigorously, the borders were measured quantitatively by fitting sigmoidal curves to intensity plots of transmitted light within layer 4 in bright-field images (Petersen and Sakmann, 2000). The border was defined as the location with the half-maximal intensity of bright field transmitted light, leaving a septal area between neighboring barrels averaging $\sim 100 \mu \mathrm{m}$ in width. The superimposed lateral limits of layer 4 barrels (Fig. $4 A$, cyan) correspond to this definition, and the evoked voltage-sensitive dye signal around the peak of the response at $12 \mathrm{msec}$ is shown with and without the barrel boundaries superimposed. The voltage-sensitive dye signal is narrowest in layer 4 and broader in both layer $2 / 3$ and layer 5 . Only small signals are detected in neighboring barrels at this time point, and, in addition, it appears that the largest drop in signal amplitude occurs at the barrel boundaries. The time dependence of the signal at different locations within layer 4 is indicated in the bottom right panel of Figure $4 A$. Large signals are detected within the layer 4 barrel with nearly equal amplitude across the barrel. The signal in the septum dividing neighboring barrels is strongly reduced in amplitude, and only small signals are detected in the neighboring barrel. The largest signal in the neighboring barrel appears after a significantly longer latency. Averaged over many experiments $(n=11)$, the latency for half-maximal signals in the stimulated barrel was $2.0 \pm 0.2 \mathrm{msec}$; for layer $2 / 3$, latency was $4.0 \pm 0.2 \mathrm{msec}$, and for the nearest neighbor layer 4 barrel, the latency measured $10.6 \pm 3.2 \mathrm{msec}$ (measured at the same distance from the stimulation electrode as the layer $2 / 3$ latency). The small signal that can be detected in neighboring barrels thus presumably results from polysynaptic excitation. The spatial extent of voltage-sensitive dye signals at $12 \mathrm{msec}$ after stimulation normalized across experiments $(n=11)$ and aligned such that the origin occurs at the barrel boundary (Fig. $4 B$ ) indicates a sharp drop in amplitude at the septum. A sigmoidal curve fitted to the data has a half-maximal value at $1 \mu \mathrm{m}$ inside the home barrel and $80 \%-$ $20 \%$ transition length of $101 \mu \mathrm{m}$. Also indicated in Figure $4 B$ are the corresponding measurements for the lateral spread of voltagesensitive dye signals in layer $2 / 3$ at $12 \mathrm{msec}$ after the stimulus. The voltage-sensitive dye signal in layer $2 / 3$ also drops in amplitude near the barrel border but it is shallower, and, importantly, $>10 \%$ of the maximal signal spreads $200 \mu \mathrm{m}$ outside the stimulated barrel (sigmoidal fit to the data gives a half-maximal value at 10 $\mu \mathrm{m}$ outside the home barrel and $80 \%-20 \%$ transition length of $131 \mu \mathrm{m})$. Thus, within layer $2 / 3$, there is considerable overlap of voltage-sensitive dye signals from neighboring barrel columns but very little within the barrels of layer 4 . Additional evidence that the determinant of the width of the voltage-sensitive dye signal in layer 4 is the anatomical barrel structure comes from the linear dependence between the bright-field barrel width and the width
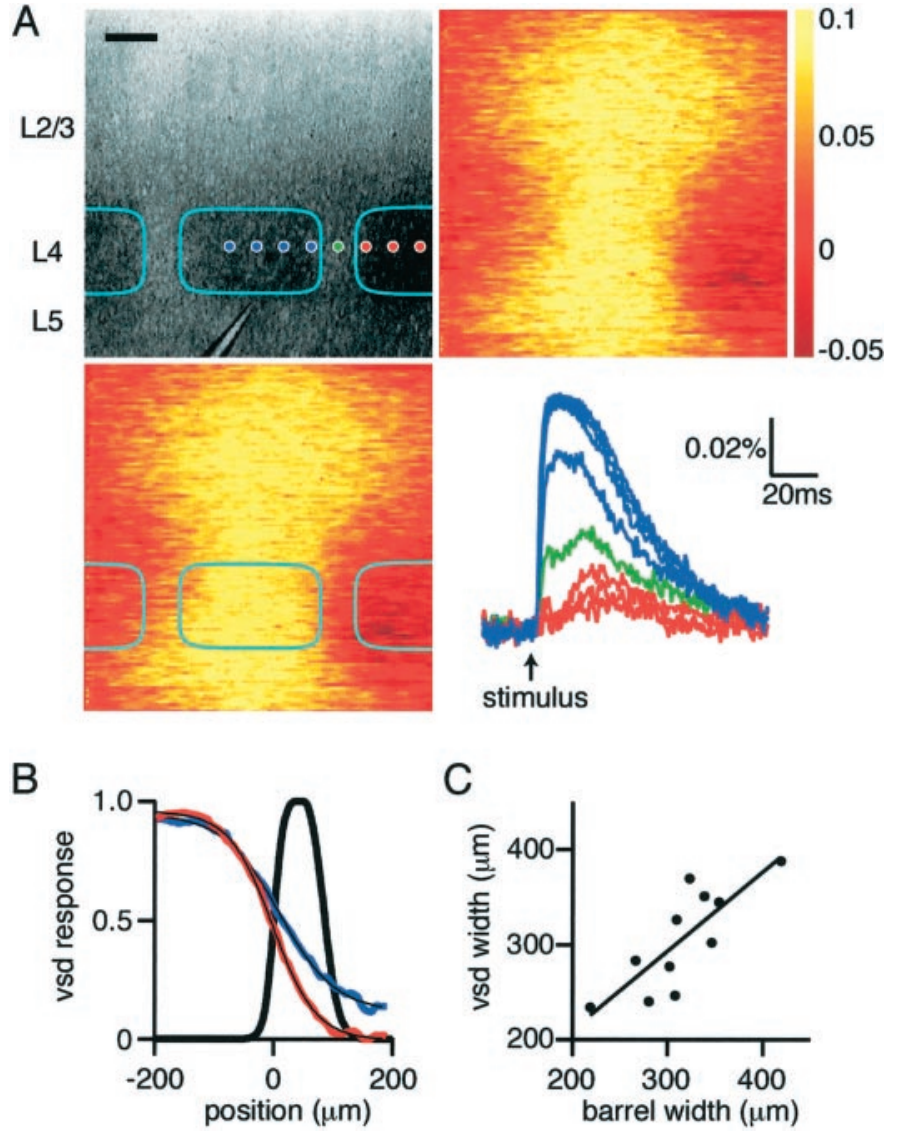

Figure 4. Voltage-sensitive dye responses within layer 4 are primarily confined to the stimulated barrel and associated cortical column. $A$, Bright-field micrograph (top left) of the region of barrel cortex imaged with the voltage-sensitive dye. Cyan boxes indicate extent of individual barrels, and the stimulation electrode is visible in the center of the barrel at the layer $4 / 5$ border. Images of the peak voltage-sensitive dye signal (12 msec after stimulation) without (top right) and with (bottom left) barrel demarcation. The optical responses at the locations indicated by dots in the top left panel are plotted (bottom right), with responses from within the stimulated barrel colored blue, from the septum in green, and from the neighboring barrel in red. The spatial extent of the response decays rapidly outside of the stimulated barrel, with very little response at short latencies detected in neighboring barrels. A small response can, however, be detected late in the neighboring barrels. Scale bar, $100 \mu \mathrm{m}$. B, The normalized spatial extent of the optical dye signal within layer 4 (red curve) measured at $12 \mathrm{msec}$ after stimulation is confined to the stimulated barrel (barrel septum shown in black, with half-maximal barrel boundary at $0 \mu \mathrm{m})$. The black superimposed curve is a sigmoidal fit, with halfmaximal value at $-1.09 \mu \mathrm{m}$ and a length constant of $36.4 \mu \mathrm{m}$. The spatial extent of the associated signals in layer $2 / 3$ is plotted in blue, indicating that, although the response is more diffuse, it also shows a marked decay over similar distances with half-maximal value at $9.85 \mu \mathrm{m}$ and a transition length of $47.3 \mu \mathrm{m}$. However, $>10 \%$ of the signal remains $200 \mu \mathrm{m}$ outside of the stimulated barrel. $C$, The full-width at half-maximum of the voltage-sensitive dye signal shows a close correlation with the anatomically defined width of the barrels stimulated.

at half-maximum of the voltage-sensitive dye signal (vsd width $=$ barrel width *0.83 $+43 ; r^{2}=0.64 ; n=11$ ) (Fig. $4 C$ ).

To resolve the lateral borders of barrels in two dimensions, tangential slices containing layer 4 were prepared following the description of Fleidervish et al. (1998). These slices allow the lateral extent of voltage-sensitive dye signals to be correlated in two dimensions with the barrel structure. In particular, it appeared to be a stringent test of our hypothesis that the global shape of the voltage-sensitive dye signal at times when it is largest 

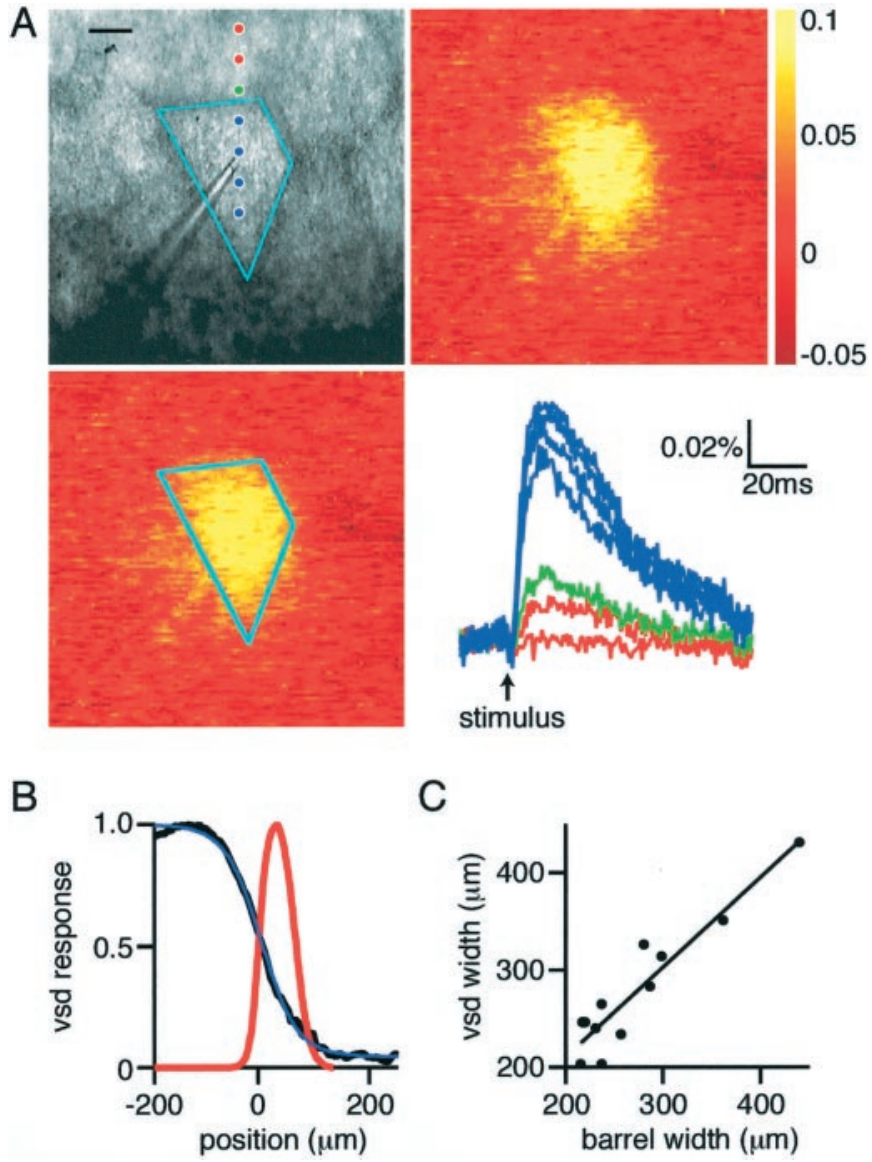

Figure 5. Barrel cortex slices cut tangential to the pia containing only layer 4 indicate that excitation is confined to the stimulated barrel. $A$, Bright-field micrograph (top left) of the region of tangentially sliced barrel cortex imaged with the voltage-sensitive dye. Cyan outline indicates the extent of the stimulated barrel with the stimulation electrode visible in the center. Images of the peak voltage-sensitive dye signal (12 msec after stimulation) without (top right) and with (bottom left) barrel demarcation. The optical responses at the locations indicated by dots in the top left panel are plotted (bottom right), with responses from within the stimulated barrel colored blue, from the septum in green, and from the neighboring barrel in red. The spatial extent of the response decays rapidly outside of the stimulated barrel with very little in neighboring barrels. The shape of the optical dye response matches closely the shape of the barrel viewed in bright-field microscopy. Scale bar, $100 \mu \mathrm{m}$. B, Quantification of the spatial extent of the voltage-sensitive dye signal with respect to the barrel border at $0 \mu \mathrm{m}$ (red line indicates septum). The voltage-sensitive dye signal is fitted with a sigmoidal curve, with half-maximal value at $6 \mu \mathrm{m}$ and length constant $32 \mu \mathrm{m}$. $C$, The full-width at half-maximum of the voltagesensitive dye signal shows a close correlation with the width of the stimulated barrels, as defined by bright-field microscopy.

should match that of the anatomical barrel structure. Barrels can have many shapes and sizes, and, in all cases tested, the outline of the barrel in the bright-field image could also be recognized in the voltage-sensitive dye signal at its largest size $(n=12)$ (an example is shown in Fig. 5A). Unlike paracoronal slices, the small response that can be detected in neighboring barrels does not occur with a much longer latency to the main response in the stimulated barrel (Fig. 5A), which could result from the inclusion of a small part of layer $2 / 3$ or layer 5 in the tangential slice preparation. However, the major decrease in the spatial profile of the voltage-sensitive dye signal was again observed to occur at the barrel boundaries (Fig. $5 B$, vsd response at $12 \mathrm{msec}$ after stimulation). The profile was fitted with a sigmoidal curve with a half-maximal value at $6 \mu \mathrm{m}$ outside the barrel and $80 \%-20 \%$ transition length of $89 \mu \mathrm{m}$, in close agreement with the data from the paracoronal slices. Furthermore, the width at half-maximal of the voltage-sensitive dye signal depended linearly on the width of the barrel quantified across a central region of each barrel (vsd width $=$ barrel width * $0.92+27 ; r^{2}=0.87 ; n=12$ ) (Fig. $5 C$ ).

\section{Modifying the spread of excitation}

Having defined the dynamic pattern of the spread of the predominantly subthreshold excitation in a column of the barrel cortex after single stimuli under control conditions, we investigated possible ways of altering the spatiotemporal spread of excitation.

\section{Role of GABAergic inhibition in limiting spatiotemporal spread of voltage-sensitive dye signals}

Inhibition mediated by synaptic release of GABA could be responsible for the limited lateral extent of excitation in a barrel column. To test this hypothesis, $\mathrm{GABA}_{\mathrm{A}}$ receptor-mediated inhibition was blocked by addition of $10 \mu \mathrm{M}$ bicuculline to the superfusate, greatly enhancing both the voltage-sensitive dye signal and EPSP amplitude in whole-cell recordings (Figs. 6, 7). During bicuculline application, voltage-sensitive dye signals were larger, lasted longer, and spread over a larger cortical region, in accord with the effects of bicuculline observed with voltagesensitive dyes in vitro (Laaris et al., 2000) and in vivo during whisker stimulation (London et al., 1989). Simultaneous wholecell voltage recordings indicated that amplitude and duration increased in a manner well correlated to the changes in optically detected signals, for example, when comparing the local voltagesensitive dye response and the membrane potential of the layer $2 / 3$ neuron (Fig. $6 B, C$ ). In the presence of bicuculline, APs are observed in whole-cell recordings from neurons in both layer 4 and layer $2 / 3$ (Fig. 6D), which was not the case in control conditions. Many APs were observed in response to single stimuli in both layer $2 / 3$ and layer 4 excitatory neurons, but the membrane potential always returned to the prestimulus value, even with 10 $\mu \mathrm{M}$ bicuculline present. Epileptic activity between stimuli was not observed, and sequential stimuli evoked reproducible responses (Fig. 6D). The absence of spontaneous activity and the ability of the membrane potential to recover its resting value quickly after stimulation might suggest that some inhibition remains despite the presence of $10 \mu \mathrm{M}$ bicuculline. This could result from the activation of $\mathrm{GABA}_{\mathrm{B}}$ receptors or ionotropic GABA receptors with low bicuculline sensitivity during massive excitation.

The initial spatiotemporal pattern of the voltage-sensitive dye response was similar in control and bicuculline (Fig. 6A). The response was initially confined to the layer 4 barrel and then spread in a columnar manner into layer $2 / 3$. Under control conditions, the response then decays, remaining columnar (as also shown in Fig. 3), whereas in the presence of bicuculline, the response increases several-fold and propagates laterally over a large region in layer $2 / 3$. The infragranular region of layer 5 , which in many cases is only weakly excited by field stimulation under control conditions, is also strongly activated by identical stimuli but in the presence of bicuculline (Fig. 6A,B). Such increases in spatial spread of voltage-sensitive dye signals are not observed in layer 4 (Fig. 7). In paracoronal slices $(n=10)$, the width at half-maximal signal amplitude is increased only by $41 \pm$ $6 \%$ within layer 4 , whereas in layer $2 / 3$, the half-width increases by at least $117 \pm 15 \%$ (quantified at peak response amplitudes). This number is likely to be an underestimate because, in many experiments (like the one shown in Fig. 7B; control response at $12 \mathrm{msec}$ 


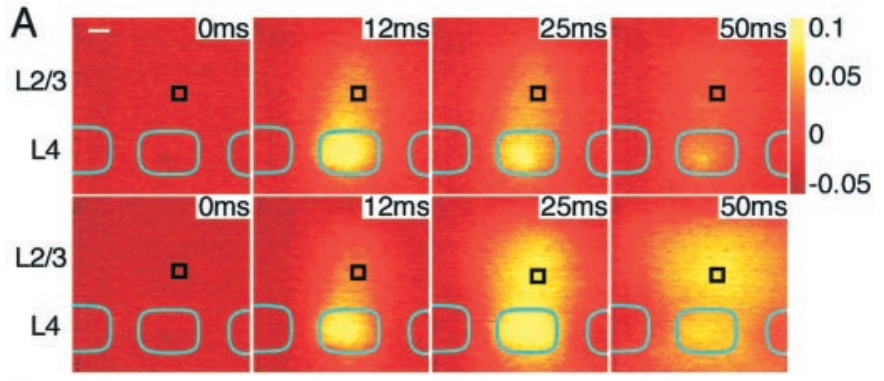

B

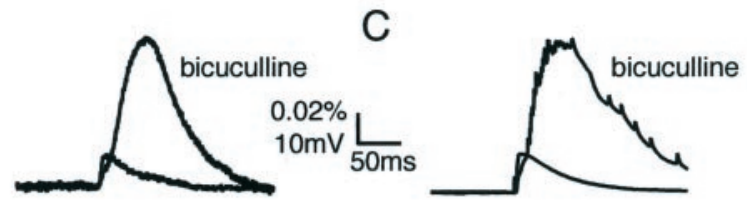

D

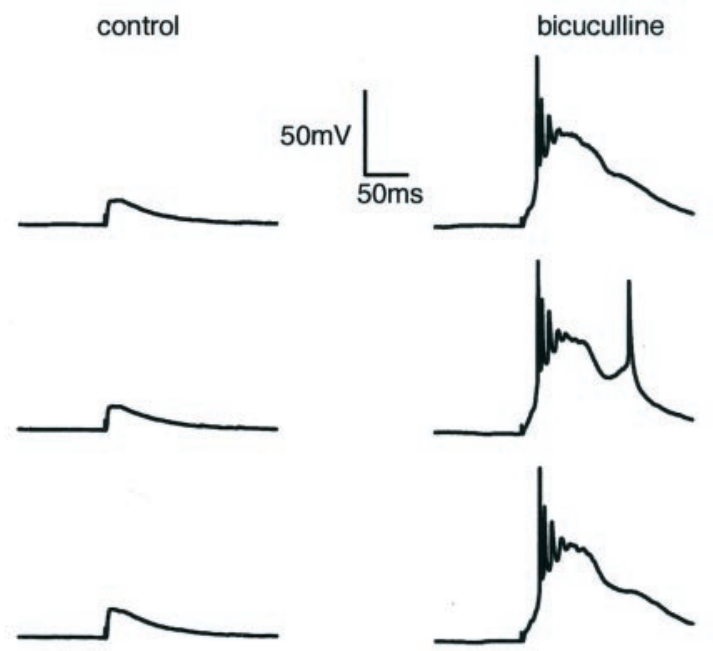

Figure 6. Block of inhibition by bicuculline evokes equivalent enhancement of whole-cell electrophysiological and voltage-sensitive dye responses. $A$, Time series of optical responses to stimulation of a layer 4 barrel under control conditions (top) or after application of $10 \mu \mathrm{M}$ bicuculline to block $\mathrm{GABA}_{\mathrm{A}}$ receptors (below). The same stimulation strength evoked a larger response with a longer duration in the presence of bicuculline. Scale bar, $100 \mu \mathrm{m}$. $B$, The effect of bicuculline on the response measured by voltage-sensitive dye in a region $(60 \times 60 \mu \mathrm{m})$ surrounding a whole-cell recording (black box in $A$ ). $C$, The effect of bicuculline on the responses measured with whole-cell recording. These traces are averages of the same 16 consecutive sweeps as for the voltage-sensitive dye measurements in $A$ and $B$. There is a close correspondence of the changes in amplitude and time course of responses with those of the voltage-sensitive dye. $D$, Consecutive individual sweeps of the whole-cell recordings under control conditions (left) and after application of bicuculline (right). No action potentials are evoked in control conditions, whereas several are evoked by each stimulus when inhibition is blocked. The responses to consecutive stimuli are similar.

after stimulation; response in the presence of bicuculline at 50 $\mathrm{msec})$, the entire field of view $(780 \times 780 \mu \mathrm{m})$ of layer $2 / 3$ is homogeneously excited and, for quantification purposes, the halfwidth of such signals was given a value of the field of view, although its real value would be larger. The result of this nonuniform propagation of excitation is that the neighboring barrels in layer 4 appear to be uniquely spared. The restricted lateral spread of excitation in layer $2 / 3$ under control conditions thus appears to result from inhibition, whereas the restriction of excitation within layer 4 to the stimulated barrel appears to result from a lack of excitatory connections between barrels. This view

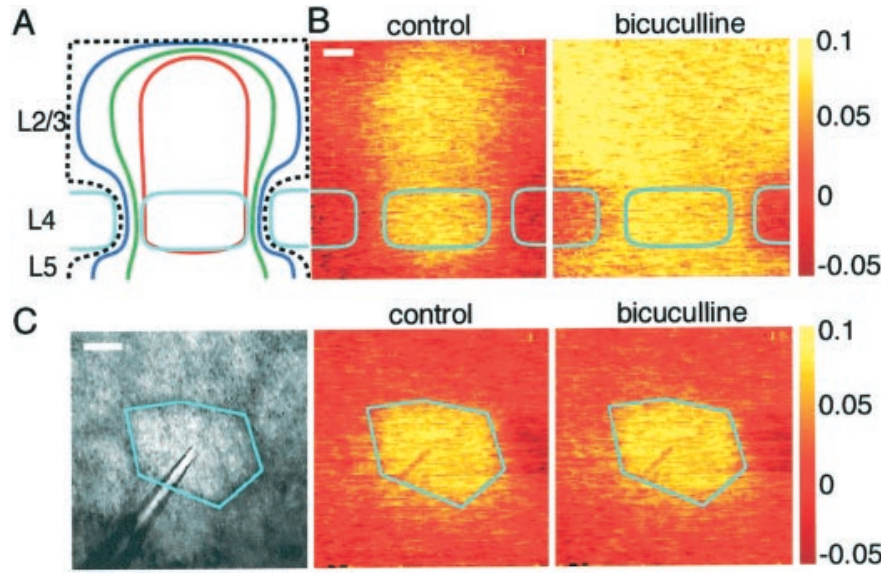

Figure 7. Block of inhibition by bicuculline potentiates the spread of excitation in $\mathrm{L} 2 / 3$ and $\mathrm{L} 5$ but not layer 4 . $A$, Schematic representation of the spread of excitation in paracoronal slices at 10 (red), 15 (green), and 20 (blue) msec after stimulation. The black dashed line indicates that excitation in layer $2 / 3$, and layer 5 often spread beyond the field of view. The spread of voltage-sensitive dye signal within layer 4 increases only a little compared with the expansion of excitation within layer $2 / 3$ and layer 5. $B$, Individual example showing columnar excitation under control conditions (image at $12 \mathrm{msec}$ ) but large lateral spread of excitation in layer $2 / 3$ and layer 5 after $10 \mu \mathrm{M}$ bicuculline application (image at $50 \mathrm{msec}$ ). Scale bar, $100 \mu \mathrm{m}$. $C$, In tangential layer 4 slices, application of bicuculline has no obvious effect on the spatial extent of excitation detected by optical imaging (control image at $12 \mathrm{msec}$; bicuculline image at $40 \mathrm{msec}$ ). Scale bar, $100 \mu \mathrm{m}$.

is in good agreement with anatomical reconstruction of dendritic and axonal arborizations of layer 4 neurons remaining confined to a single barrel (Fig. 2). The lateral propagation of excitation within layer $2 / 3$ when inhibition is blocked presumably reflects the suprathreshold activity of layer $2 / 3$ neurons, with their axons projecting across to neighboring barrel columns exciting other layer $2 / 3$ pyramidal neurons and also contacting the apical layer 5 pyramidal dendrites, thus evoking excitation within layer 5. Furthermore, it would appear that neurons of layer $2 / 3$ and layer $5 / 6$ only weakly excite the barrel of layer 4 sandwiched in between them.

We further tested the effect of bicuculline on tangential layer 4 slices to see whether significant extension of stimulated areas could be evoked. After application of bicuculline, the area contained within a half-maximal contour increased by only $9 \pm 12 \%$ ( $n=10$; quantified at peak response amplitudes). An example experiment showing a hexagonally shaped barrel, with a similarly shaped voltage-sensitive dye signal, which was barely affected by bicuculline application, is shown in Figure $7 C$ (control response at $12 \mathrm{msec}$ after stimulation; response in the presence of bicuculline at $40 \mathrm{msec}$ ). The smaller effect of bicuculline in tangential slices of layer 4 compared with the effect within layer 4 of paracoronal slices presumably results from the lack of excitation from layer $2 / 3$ and layer $5 / 6$ in these tangential slices. These data suggest that, in layer 4 , the barrels are functionally independent even when enormous activity is evoked in all neighboring neocortical regions.

\section{Repetitive $10 \mathrm{~Hz}$ stimulation does not alter the spatiotemporal extent of voltage-sensitive dye signals}

During active exploration, rodents move their whiskers rhythmically at a frequency of $\sim 10 \mathrm{~Hz}$, which, it is thought, increases the sampling rate of nearby space, allowing objects to be rapidly and accurately located. It is thus of particular interest to investigate the effects of repetitive stimulation on the spatiotemporal signal- 
ing patterns within barrel cortex. When a barrel is stimulated at $10 \mathrm{~Hz}$ frequency, each stimulus evokes a clear response, which is similar to the response to the first stimulus. However, during the $10 \mathrm{~Hz}$ train of 10 stimuli, the response amplitude decreases to $54 \pm 7 \%$ within layer 4 and to $62 \pm 6 \%$ in layer $2 / 3(n=13)$. More importantly, however, the spatial structure of the voltage-sensitive dye responses remains almost unaltered, with only slight increases in the half-width of the evoked excitation measured $12 \mathrm{msec}$ after stimuli comparing first and last responses from $387 \pm 23$ to $437 \pm 46 \mu \mathrm{m}$ in layer 4 and $479 \pm 43$ to $509 \pm 56 \mu \mathrm{m}$ in layer $2 / 3$, with a mean layer 4 barrel width of $399 \pm 21 \mu \mathrm{m}(n=13)$. The functional columns of excitation observed with single stimuli are thus preserved during repetitive stimulation but with smaller signal amplitudes. This may be attributable to a depression of excitatory synaptic transmission and/or increased inhibition.

\section{Simultaneous stimulation of neighboring barrels has only a weak nonlinear effect on the spatiotemporal extent of voltage- sensitive dye signals}

For the rodent to gather information concerning nearby objects, it is likely that the information concerning the movement of a single whisker is processed in concert with that from neighboring whiskers and indeed the entire whisker array (Moore et al., 1999). One might therefore expect substantial modifications of the response of a barrel column depending on whether or not its neighbor is stimulated or not. Indeed, in vivo both supralinear and sublinear changes to responses from one whisker have been observed by pairing stimulation with neighboring whiskers (Simons, 1985; Kleinfeld and Delaney, 1996; Ghazanfar and Nicolelis, 1997; Shimegi et al., 2000). To address whether such interactions also occur in vitro in the neocortex, we stimulated neighboring barrel columns simultaneously and compared this with the sum of the responses to stimulation of the barrel columns separately. The results suggested that responses are summated almost linearly (Fig. 8). To quantify the effect, the half-width of signals for layer 4 and layer $2 / 3$ for the experimental simultaneous stimulation and the computed sum of individual responses were compared at $12 \mathrm{msec}$ after stimulation. The width of excitation in layer 4 did not change significantly (experiment, $534 \pm 40 \mu \mathrm{m}$; linear sum, $526 \pm 40 \mu \mathrm{m}$; paired $t$ test, $p>0.4 ; n=12$ ), whereas the width in layer $2 / 3$ showed a slight superlinearity, with an experimental width of $722 \pm 43 \mu \mathrm{m}$ compared with the expected value of $676 \pm 41 \mu \mathrm{m}$ (paired $t$ test, $p<0.005 ; n=12$ ). Responses in layer 4 barrels show little spatial overlap with nearest neighbors, and the combined response is entirely linear. Excitation in layer $2 / 3$ extends well into the neighboring barrel column, but nonetheless the response is summated close to linearly. The small supralinear interaction component within layer $2 / 3$ could result from supralinear summation of subthreshold EPSPs via voltagegated channels.

\section{NMDAR-dependent expansion of the spread of excitation can be induced by pairing high-frequency stimuli}

Both the development and plasticity of the neocortex have been suggested to be regulated in part by electrical activity-dependent processes. Changes in synaptic efficacy of inputs onto layer $2 / 3$ pyramidal neurons have been proposed recently to underlie some of the major changes in receptive fields induced by alterations in sensory experience (Diamond et al., 1994; Finnerty et al., 1999; Feldman, 2000). To investigate whether expansion of the spatial extent of excitation in layer $2 / 3$ can be observed in vitro, we stimulated neighboring barrels simultaneously using a pairing

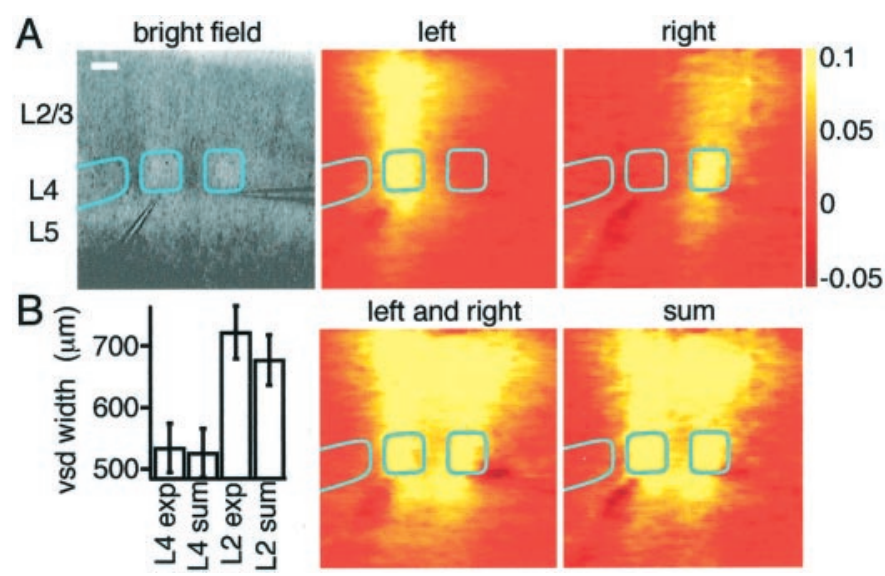

Figure 8 . Activity evoked by the simultaneous stimulation of neighboring barrels is summated linearly. $A$, Bright-field micrograph showing stimulation electrodes placed in the lower center of neighboring barrels. Stimulation of single barrels (left or right) evoked responses in the barrel column. The response to simultaneous stimulation of neighboring barrels (labeled left and right) appears to be equivalent to the simple sum (labeled sum) of the responses evoked by stimulation of the barrels separately. The voltage-sensitive dye images are shown at $12 \mathrm{msec}$ after stimulus. Scale bar, $200 \mu \mathrm{m}$. B. Comparison of the full-width at half-maximal for the voltage-sensitive dye signals between the experimental simultaneous stimulation of neighboring barrels and the linear sum of the individual responses. In layer 4, the width of excitation is unchanged and only a small increase of width is detected in layer $2 / 3$.

protocol similar to one that causes NMDA receptor-dependent potentiation between layer $2 / 3$ pyramidal neurons (Egger et al., 1999). Neighboring barrels were stimulated simultaneously every $5 \mathrm{sec}$ with a train of five stimuli separated by $50 \mathrm{msec}$ for a total of 100 stimuli separated into 20 bursts. The effects of this pairing procedure were measured 10-15 min later, well beyond the period of short-term plasticity or posttetanic potentiation. Each barrel was subsequently stimulated separately, and the spatial extent of the evoked voltage-sensitive dye signal was compared with the prepairing response. There was a no obvious change to the spatial extent of the voltage-sensitive dye signal in layer 4. In some experiments, there appeared to be a clear enhancement of the response in layer $2 / 3$ above the neighboring barrel with which it had shared the pairing stimulation, but there was no significant change when all experiments were included (paired $t$ test, $p=$ $0.076 ; n=16)$.

In view of the relatively small supralinear effect in layer $2 / 3$ of stimulating neighboring barrels (Fig. 8), it is likely that this stimulation protocol causes only a weak coincidence (pairing) of activity in layer $2 / 3$ pyramidal neurons. To evoke a stronger pairing of excitation in layer $2 / 3$ neurons, the stimulation of a barrel in layer 4 was paired with simultaneous stimulation of a layer $2 / 3$ region in the neighboring barrel column. One stimulation electrode was placed in the lower center of a barrel and evoked excitation limited to a barrel column as described previously (Fig. 9A). The second stimulation electrode was placed in layer $2 / 3$ within $200 \mu \mathrm{m}$ of the right-hand lateral extent of the barrel column. It evoked voltage-sensitive dye signals, which were strongest in the region immediately surrounding the stimulation electrode (Fig. 9A). The same pairing protocol was used as before, and again the effects were measured 10-15 min later to avoid measuring effects of short-term plasticity or posttetanic potentiation. This pairing protocol induced a significant asymmetric expansion in the lateral extent of the layer $2 / 3$ voltage- 
A

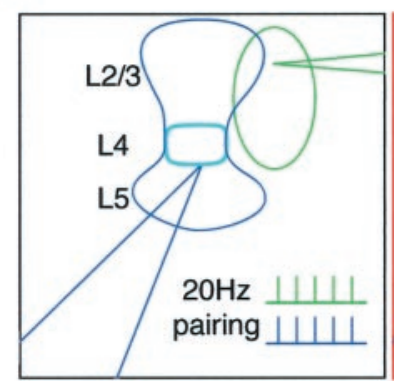

before pairing
L2/3 stimulus

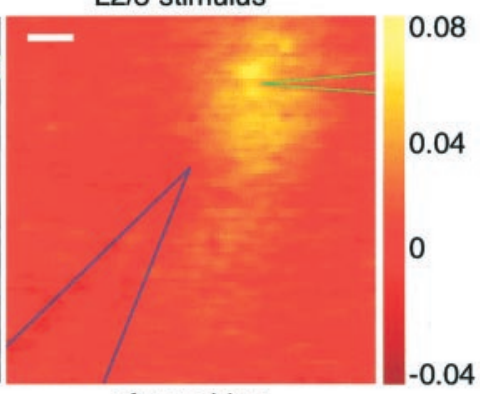

after pairing

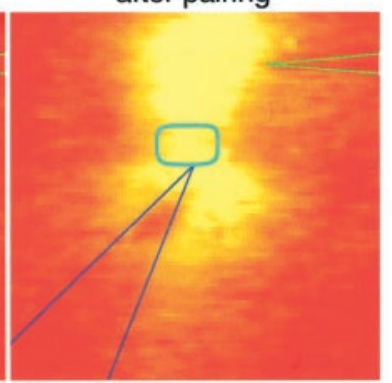

B

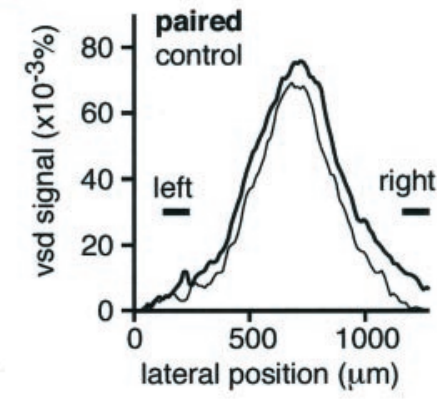

$\mathrm{D}$

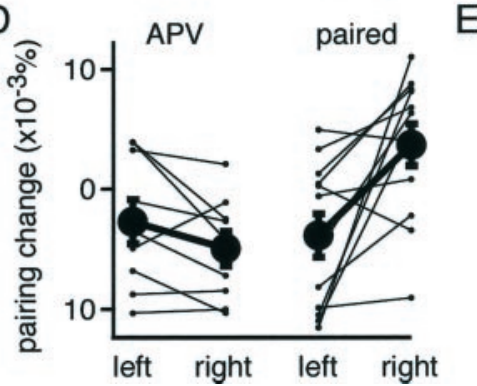

C

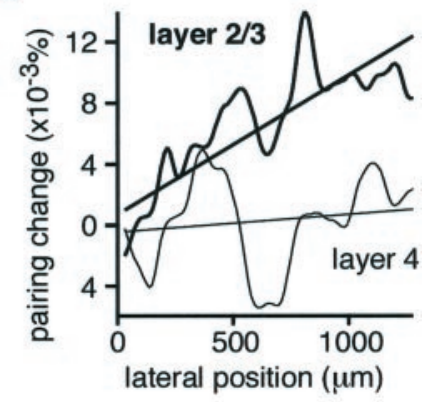

E

L2/3

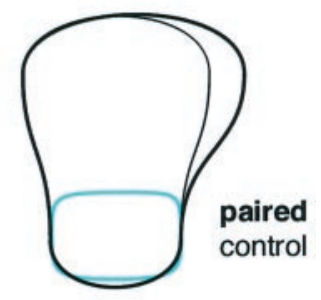

Figure 9. Pairing stimulation of a barrel column with a neighboring region of layer 2/3 induces an NMDA receptor-dependent expansion of excitation. $A$, The experimental arrangement and stimulation protocol used to induce a spatial expansion of responses (top left). Stimulation of the electrode in layer 2/3 placed to the right of the barrel column evoked a local response (top right). Stimulation of the electrode at the lower center of a barrel evoked a columnar response (bottom left). Both electrodes were then stimulated simultaneously five times at $50 \mathrm{msec}$ intervals, and this was repeated at 5 sec intervals for a total of 20 pairing bursts. Responses were then tested $15-20 \mathrm{~min}$ after this pairing protocol, and the area within layer $2 / 3$ excited by stimulating a barrel was found to have expanded toward the layer $2 / 3$ stimulating electrode located on the right (bottom right). The voltage-sensitive dye images are shown at $12 \mathrm{msec}$ after stimulus. Scale bar, $200 \mu \mathrm{m}$. B. The voltage-sensitive dye signal across the lateral extent of layer $2 / 3$ after the pairing is selectively enhanced on the right of the barrel column at which the layer $2 / 3$ stimulating electrode was located. A region at equal distance from the peak response but to the left shows no potentiation. The difference between left and right regions shows that the spatial extent of the layer $2 / 3$ signal can be asymmetrically changed. Same experiment as shown in $A$. $C$, Subtracting the lateral voltage-sensitive dye signal after pairing from the control signal shows that the signal is increased on the right after pairing within layer $2 / 3$, but within layer 4 there is no asymmetrical change. Same experiment as shown in $A$ and $B . D$, Across all experiments, the changes at equal distances to the left and to the right of the peak are plotted. On average, only a small rundown of responses is observed after pairing in the presence of APV, but under control conditions, there is a significant selective enhancement of responses to the right of the peak, which is the region close to the layer $2 / 3$ stimulating electrode. $E$, A schematic drawing of the changes induced by pairing the response of a barrel column with excitation of a neighboring region of layer $2 / 3$ on the right.

sensitive dye signal evoked by stimulation of the barrel column (Fig. 9, quantified at $12 \mathrm{msec}$ after stimulation). The amplitude of the voltage-sensitive dye signal evoked by stimulation of a layer 4 barrel is more than doubled in the region close to the stimulation electrode in layer 2/3. Quantified over an area of $200 \times 200 \mu \mathrm{m}$ located in the middle of layer $2 / 3$ and laterally $500 \mu \mathrm{m}$ from the center of the barrel column toward the stimulating electrode in layer $2 / 3$, the signal increased after pairing by $133 \pm 47 \%(n=13$; paired $t$ test, $p<0.01$ ). Measured at the same lateral distance from the spatial peak of the voltage-sensitive dye response but on the opposite side of the barrel column response, on average there is a slight decrease in the signal strength (Fig. 9D). This is probably attributable to a slight rundown of the responses, because an equal change is observed after the high-frequency pairing protocol in the presence of the NMDA receptor antagonist D-APV $(100 \mu \mathrm{M})$. However, the asymmetrical expansion of the spatial extent of the voltage-sensitive dye signal close to the layer $2 / 3$ pairing stimulation is completely blocked by D-APV $(n=9$; $p>0.1$ ). If identical stimuli are delivered but the trains of stimuli are separated by $500 \mathrm{msec}$ instead of simultaneously, then no potentiation is induced. After such unpaired protocols, the amplitude of the evoked signals decreased by $23 \pm 4 \%(n=10)$, quantified as before near the layer $2 / 3$ stimulating electrode. To further test that the potentiation of voltage-sensitive dye responses after pairing was not attributable to changes in optical properties of the slice, whole-cell recordings were made from excitatory neurons near the layer $2 / 3$ stimulating electrode. After the pairing protocol, EPSPs evoked by stimulation of the layer 4 barrel increased in amplitude by $79 \pm 44 \%(n=7)$. During the pairing protocol, action potentials were often evoked in the neuron by the simultaneous stimulation in layer 4 and layer $2 / 3$. The potentiation observed here may thus share a common mechanism with the increases in synaptic efficacy associated with EPSPs paired with action potentials evoked by somatic current injection (Markram et al., 1997; Egger et al., 1999; Feldman, 2000); however, changes in excitability or inhibition may also contribute. Thus, several molecular mechanisms could be involved in generating the expansion of the spread of excitation after the pairing protocol.

\section{DISCUSSION}

\section{Functional neocortical column}

The pattern of the spread of excitation initiated in a barrel of layer 4 provides a new, dynamic view of a neocortical column. The early response $(\sim 2 \mathrm{msec})$ to a single stimulus is limited to the stimulated barrel in layer 4 , and it is only later $(\sim 5 \mathrm{msec})$ that the close correspondence to the anatomically defined barrel column develops. This correspondence remains during the decay phase of the response, lasting $\sim 50 \mathrm{msec}$ after the stimulus. This spatiotemporal pattern of the propagation of excitation in a columnar manner also remains invariant during repetitive stimulation and 
is only weakly affected by stimulation of a neighboring barrel column. The functional confinement of the spread of excitation to a column was controlled by GABAergic inhibition. Even in the absence of inhibition, however, the initial milliseconds of the spread of excitation is unchanged, showing the stereotypical columnar propagation of the axonal projection of layer 4 excitatory neurons. Later $(\sim 25 \mathrm{msec})$ excitation spreads laterally in layer $2 / 3$ and reaches layer 5/6, presumably through recurrent excitatory synaptic transmission of pyramidal neurons.

\section{Barrels are functionally independent}

Our anatomical reconstruction of a normalized barrel is in accord with previous studies showing that both the axonal and dendritic arbors are confined to the barrel in which the soma is located (Woolsey et al., 1975; Harris and Woolsey, 1983; Simons and Woolsey, 1984; Lübke et al., 2000; Petersen and Sakmann, 2000). Mapping the connectivity of pairs of excitatory neurons within layer 4 has furthermore shown that barrels are functionally independent (Petersen and Sakmann, 2000). However, such studies were limited to the few neurons selected for whole-cell recording and cannot address what occurs when a much larger ensemble of neurons are stimulated. Voltage-sensitive dye signals show that the excitatory neuronal network of barrels in layer 4 is functionally independent, even during massive excitation evoked by stimulation in the presence of bicuculline. This not only implies that the network within each barrel is independent of neighboring barrels but also that activity in supragranular and infragranular cortical laminas are only able to weakly excite layer 4. This inability of excitation initiated in one barrel to spread into another in vitro may explain the lack of effect on unit responses to surround whisker stimulation in vivo after lesions to neighboring barrels (Goldreich et al., 1999).

\section{Spread of excitation in layer $2 / 3$}

The maximal response in layer $2 / 3$ is primarily confined to a region with a similar width to the layer 4 barrel, thus defining a "functional" barrel column. Whereas the layer 4 response shows little overlap with the neighboring barrel, the signal evoked somewhat later in layer $2 / 3$ is significantly broader $(26 \pm 4 \%)$ than the layer 4 signal. A large fraction of the lateral decay of excitation occurs close to the boundary of the barrel column, but nonetheless significant excitation is detected in layer $2 / 3$ of the neighboring barrel column. This overlap between barrel columns is also reflected in the supralinearity of the summed layer $2 / 3$ responses evoked by stimulation of two neighboring columns. We thus conclude that sensory signals from the thalamus would be processed separately in layer 4 barrels, but within layer $2 / 3$ there are significant excitatory interactions between columns. The lateral extent of excitation within layer $2 / 3$ is strongly regulated by inhibition, and thus the activity of GABAergic interneurons may determine whether the processing of whisker movement related cortical signals occurs at a local (single-column) or a global (multicolumn) scale in layer $2 / 3$.

\section{Comparison with in vivo data}

Optical imaging of voltage-sensitive dyes in vivo has yielded maps of whisker-evoked activity in somatosensory cortex (Orbach et al., 1985; Kleinfeld and Delaney, 1996). Stimulation of a single mystacial whisker can evoke voltage-sensitive dye signals over an area of several square millimeters of the cortical surface. The spread of excitation measured in vivo thus appears to be considerably more extensive than what we observe in vitro in layer $2 / 3$ from the stimulation of a single layer 4 barrel. The larger excitation area observed in vivo is likely to result from a number of factors.

The receptive field of both thalamic ventroposterior medial neurons (Ito, 1988; Simons and Carvell, 1989; Armstrong-James and Callahan, 1991; Diamond et al., 1992; Brecht and Sakmann, 2001) and layer 4 neurons (Moore and Nelson, 1998; Zhu and Connors, 1999) are considerably broader than might be expected from the apparent precision of the primary anatomical connections specifically wiring the sensory neurons of a given whisker to its homologous barrel. Thus, deflection of a single whisker evokes EPSPs and even APs in neurons located in many layer 4 barrels and septal regions. The large receptive fields of layer 4 neurons could contribute to the large spatial extent of responses in layer $2 / 3$ evoked by single whisker stimulation measured in vivo by voltage-sensitive dye. This contrasts with our selective stimulation of a single barrel allowing a simpler analysis of the functional architecture of synaptic connections.

The width of layer $2 / 3$ signals observed in vitro by stimulation of a single layer 4 barrel depended strongly on the state of inhibition. Blocking inhibition with $10 \mu \mathrm{M}$ bicuculline caused excitation to spread laterally over much larger regions of layer $2 / 3$, in closer agreement with the size of the spread of excitation observed in vivo. The balance between excitation and inhibition in vivo may thus be shifted in favor of excitation compared with in vitro. That this is the case is suggested by the much higher levels of both subthreshold and suprathreshold spontaneous activity of neurons recorded in vivo. Additional support for this explanation comes from a comparison of the duration of the voltage-sensitive dye signals in vivo and in vitro. Whereas under control conditions in vitro voltage-sensitive dye signals last $\sim 50 \mathrm{msec}$, in vivo a single deflection of a single whisker can evoke responses lasting up to $500 \mathrm{msec}$ (Kleinfeld and Delaney, 1996), suggesting that inhibition in vivo does not prevent prolonged excitation. The larger neocortical area excited in vivo as recorded by voltage-sensitive dye imaging may thus result from propagating activity within layer $2 / 3$ mediated by local recurrent excitatory collaterals of the layer $2 / 3$ pyramidal neurons. In support of such a conclusion, high temporal resolution imaging of responses to single whisker deflections result in an initial excited region that is little larger than a layer 4 barrel, but which subsequently rapidly expands to cover a much larger region over the next milliseconds (Petersen et al., 2001).

\section{NMDA receptor-dependent plasticity of barrel column responses}

Previous studies of NMDA receptor-dependent plasticity in neocortical slices have focused on the increases in local field potentials or responses in individual neurons (Kirkwood et al., 1993; Castro-Alamancos et al., 1995; Markram et al., 1997; Feldman, 2000). Because of the very localized nature of such measurements, there has been no information about changes in the spatial pattern of excitation associated with stimulation protocols that induce potentiation of individual synaptic responses. The spatial correlate of synaptic potentiation of many synapses in the ensemble neocortical circuit would be an expansion in the area excited. By imaging voltage-sensitive dye signals, we observed NMDA receptor-dependent changes in the spatial extent of excitation induced by pairing large ensembles of neurons in layer $2 / 3$. Of particular interest is the asymmetry of the spatial extension of the voltage-sensitive dye response toward the simultaneously stimulated area. Responses thus extend toward simultaneously active regions. Such large-scale alterations in the spatiotemporal profile of responses may represent an in vitro correlate of map plasticity 
induced by alterations in sensory input to the neocortex (for review, see Buonomano and Merzenich, 1998). The most obvious comparison is with studies of cortical response plasticity after whisker trimming, although this plasticity develops over a period of days, whereas the plasticity we describe here is induced within a couple of minutes and the effects were analyzed 10-15 min later. The plasticity that we describe might thus represent the early steps induced by whisker trimming protocols, which leave only two whiskers intact. In these so-called "whisker pairing" experiments, the response of neurons in one spared barrel to stimulation of the neighboring spared whisker are greatly potentiated (Diamond et al., 1994). Such changes are in accord with the data presented here. If neighboring regions are stimulated strongly in the absence of other input, then the functional barrel column will extend toward the simultaneously active region in layer $2 / 3$. That changes in the spatial extent of voltage-sensitive dye signals can be induced by pairing protocols suggests that voltage-sensitive dye imaging may also be useful to assess in vivo plasticity of cortical maps induced by alterations in sensory experience.

\section{REFERENCES}

Agmon A, Connors BW (1991) Thalamocortical responses of mouse somatosensory (barrel) cortex in vitro. Neuroscience 41:365-380.

Armstrong-James M, Callahan CA (1991) Thalamocortical processing of vibrissal information in the rat. II. Spatiotemporal convergence in the thalamic ventroposterior medial nucleus (VPM) and its relevance to generation of receptive fields of S1 cortical "barrel" neurons. J Comp Neurol 303:211-224.

Armstrong-James M, Fox K, Das-Gupta A (1992) Flow of excitation within rat barrel cortex on striking a single vibrissa. J Neurophysiol 68:1345-1358.

Bernardo KL, McCasland JS, Woolsey TA, Strominger RN (1990) Local intra- and interlaminar connections in mouse barrel cortex. J Comp Neurol 291:231-255.

Brecht M, Sakmann B (2001) Whisker maps of neuronal subclasses in the rat ventral posterior medial (VPM) thalamus identified by wholecell voltage recording and morphological reconstruction. J Physiol (Lond), in press.

Buonomano DV, Merzenich MM (1998) Cortical plasticity: from synapses to maps. Annu Rev Neurosci 21:149-186.

Castro-Alamancos MA, Donoghue JP, Connors BW (1995) Different forms of synaptic plasticity in somatosensory and motor areas of neocortex. J Neurosci 15:5324-5333.

Diamond ME, Armstrong-James M, Ebner FF (1992) Somatic sensory responses in the rostral sector of the posterior group (POm) and in the ventral posterior medial nucleus (VPM) of the rat thalamus. J Comp Neurol 318:462-476.

Diamond ME, Huang W, Ebner FF (1994) Laminar comparison of somatosensory cortical plasticity. Science 265:1885-1888.

Durham D, Woolsey TA (1977) Barrels and columnar cortical organization: evidence from 2-deoxyglucose (2-DG) experiments. Brain Res 137:168-174.

Egger V, Feldmeyer D, Sakmann B (1999) Coincidence detection and changes of synaptic efficacy in spiny stellate neurons in rat barrel cortex. Nat Neurosci 2:1098-1105.

Feldman DE (2000) Timing-based LTP and LTD at vertical inputs to layer II/III pyramidal cells in rat barrel cortex. Neuron 27:45-56.

Feldmeyer D, Silver RA, Lübke J, Sakmann B (1999) Synaptic connections between spiny layer 4 neurones and layer $2 / 3$ pyramidal cells of rat barrel cortex. J Physiol (Lond) 518P:141P.

Finnerty GT, Roberts LS, Connors BW (1999) Sensory experience modifies the short-term dynamics of neocortical synapses. Nature 400:367-371.

Fleidervish IA, Binshtok AM, Gutnick MJ (1998) Functionally distinct NMDA receptors mediate horizontal connectivity within layer 4 of mouse barrel cortex. Neuron 21:1055-1065.

Ghazanfar AA, Nicolelis MAL (1997) Nonlinear processing of tactile information in the thalamocortical loop. J Neurophysiol 78:506-510.

Goldreich D, Kyriazi HT, Simons DJ (1999) Functional independence of layer IV barrel in rodent somatosensory cortex. J Neurophysiol 82:1311-1316.

Harris RM, Woolsey TA (1983) Computer-assisted analyses of barrel neuron axons and their putative synaptic contacts. J Comp Neurol 220:63-79.

Higashi S, Crair MC, Kurotani T, Inokawa H, Toyama K (1999) Altered spatial patterns of functional thalamocortical connections in the barrel cortex after neonatal infraorbital nerve cut revealed by optical recording. Neuroscience 91:439-452.

Ito M (1988) Response properties and topography of vibrissa-sensitive VPM neurons in the rat. J Neurophysiol 60:1181-1197.

Jensen KF, Killackey HP (1987) Terminal arbors of axons projecting to the somatosensory cortex of the adult rat. I. The normal morphology of specific thalamocortical afferents. J Neurosci 7:3529-3543.

Kirkwood A, Dudek SM, Gold JT, Aizenman CD, Bear MF (1993) Common forms of synaptic plasticity in hippocampus and neocortex in vitro. Science 260:1518-1521.

Kleinfeld D, Delaney KR (1996) Distributed representation of vibrissa movement in the upper layers of somatosensory cortex revealed with voltage-sensitive dyes. J Comp Neurol 375:89-108.

Kossut M, Hand PJ, Greenberg J, Hand CL (1988) Single vibrissal cortical column in SI cortex of rat and its alterations in neonatal and adult vibrissa-deafferented animals: a quantitative $2 \mathrm{DG}$ study. J Neurophysiol 60:829-852.

Laaris N, Carlson GC, Keller A (2000) Thalamic-evoked synaptic interactions in barrel cortex revealed by optical imaging. J Neurosci 20:1529-1537.

London JA, Cohen LB, Wu JY (1989) Optical recordings of the cortical response to whisker stimulation before and after the addition of an epileptogenic agent. J Neurosci 9:2182-2190.

Lorente de Nó R (1992) The cerebral cortex of the mouse (a first contribution: the "acoustic" cortex) (Fairén A, Regidor J, Kruger L, translators). Somatosens Mot Res 9:3-36.

Lübke J, Egger V, Sakmann B, Feldmeyer D (2000) Columnar organization of dendrites and axons of single and synaptically coupled excitatory spiny neurons in layer 4 of the rat barrel cortex. J Neurosci 20:5300-5311.

Markram H, Lübke J, Frotscher M, Sakmann B (1997) Regulation of synaptic efficacy by coincidence of postsynaptic APs and EPSPs. Science 275:213-215.

Masino SA, Frostig RD (1996) Quantitative long-term imaging of the functional representation of a whisker in rat barrel cortex. Proc Natl Acad Sci USA 93:4942-4947.

McCasland JS, Woolsey TA (1988) New high-resolution 2-deoxyglucose method featuring double labeling and automated data collection. J Comp Neurol 278:543-554.

Moore CI, Nelson SB (1998) Spatio-temporal subthreshold receptive fields in the vibrissa representation of rat primary somatosensory cortex. J Neurophysiol 80:2882-2892.

Moore CI, Nelson SB, Sur M (1999) Dynamics of neuronal processing in rat somatosensory cortex. Trends Neurosci 22:513-520.

Orbach HS, Cohen LB, Grinvald A (1985) Optical mapping of electrical activity in rat somatosensory and visual cortex. J Neurosci 5:1886-1895.

Petersen CCH, Sakmann B (2000) The excitatory neuronal network of rat layer 4 barrel cortex. J Neurosci 20:7579-7586.

Petersen CCH, Grinvald A, Sakmann B (2001) Spatiotemporal dynamics of barrel cortex responses imaged in vitro and in vivo. Soc Neurosci Abstr 27:337.

Petersen RS, Diamond ME (2000) Spatial-temporal distribution of whisker-evoked activity in rat somatosensory cortex and the coding of stimulus location. J Neurosci 20:6135-6143.

Shimegi S, Akasaki T, Ichikawa T, Sato H (2000) Physiological and anatomical organization of multiwhisker response interactions in the barrel cortex of rats. J Neurosci 20:6241-6248.

Simons DJ (1985) Temporal and spatial integration in the rat S1 vibrissa cortex. J Neurophysiol 54:615-635.

Simons DJ, Carvell GE (1989) Thalamocortical response transformation in the rat vibrissa/barrel system. J Neurophysiol 61:311-330.

Simons DJ, Woolsey T (1984) Morphology of Golgi-Cox-impregnated barrel neurons in rat SmI cortex. J Comp Neurol 230:119-132.

Woolsey TA, Van der Loos H (1970) The structural organisation of layer IV in the somatosensory region (SI) of the mouse cerebral cortex: the description of a cortical field composed of discrete cytoarchitectonic units. Brain Res 17:205-242.

Woolsey TA, Dierker ML, Wann DF (1975) Mouse SmI cortex: qualitative and quantitative classification of Golgi-impregnated barrel neurons. Proc Natl Acad Sci USA 72:2165-2169.

Yuste R, Tank DW, Kleinfeld D (1997) Functional study of the rat cortical microcircuitry with voltage-sensitive dye imaging of neocortical slices. Cereb Cortex 7:546-558.

Zhu JJ, Connors BW (1999) Intrinsic firing patterns and whiskersevoked synaptic responses of neurons in the rat barrel cortex. J Neurophysiol 81:1171-1183. 